\title{
La cobertura sanitaria de un ejército en retirada: la actuación de un Capitán Médico durante la Batalla del Maestrazgo (abril-julio de 1938)
}

\author{
García Ferrandis X. ${ }^{1}$
}

Sanid. mil. 2012; 68 (3): 187-192; ISSN: 1887-8571

\begin{abstract}
RESUMEN
Antecedentes y objetivos: Tras la entrada definitiva de las tropas del general Franco en la ciudad de Teruel en febrero de 1938 se inició la retirada progresiva del Ejército de Maniobra de la república. El rápido avance de las fuerzas nacionales hacia el Mediterráneo marcó las características de la cobertura sanitaria militar. El objetivo de este trabajo es realizar una aproximación a la Sanidad del Ejército republicano durante su retirada por el Maestrazgo castellonense, que tuvo lugar entre abril y julio de 1938 en el contexto de la Guerra Civil Española (1936-1939). Material y métodos: Hemos utilizado una fuente de gran valor histórico, como es el testimonio oral de un Capitán Médico del Ejército de la república. Los datos históricos que nos ha aportado han sido pertinentemente corroborados y complementados por fuentes de bibliografía secundaria. Asimismo, se han analizado documentos del Archivo de la Excma. Diputación Provincial de Valencia. Resultados: El embate de las tropas nacionales durante la Batalla del Maestrazgo hizo que las infraestructuras sanitarias republicanas tuvieran que replegarse con gran rapidez. Conclusiones: Se establece una relación entre la inestabilidad del frente de guerra y la organización Sanidad Militar.
\end{abstract}

PALABRAS CLAVE: Guerra Civil Española, Sanidad Militar, ejército en retirada, Batalla del Maestrazgo.

\section{Health coverage for a retreating army: a Medical Captain's action during the Maestrazgo campaign (April-July 1938)} SUMMARY

Precedents and purposes: After Franco's troops entered the city of Teruel in February 1938, the Maneuver Army, one of the best prepared in the Republican Popular Army, started its progressive retreat. The rapid advance of national troops toward the Mediterranean sea marked the military health care characteristics. This paper analyses the Maneuver Army's Health during its retreat along the Maestrazgo of Castellón, which took place between April and June 1938 in the context of the Spanish Civil War (1936-1939). Material and method: We have used a great historic value source, a former Republican Army Medical Captain's oral testimony. Historical data have been appropriately collaborated and complemented by secondary bibliographical sources. Likewise, documents from the Archives of the Provincial Council of Valencia have been consulted. Results: High mobility and ubiquity characterized medical classification posts and frontline Republican hospitals during the Maestrazgo campaign, because enemy's onslaught made these health infrastructure to retreat rapidly. Conclusions: A relation between war front's instability and military health characteristics is established.

KEY WORDS: Spanish Civil War, Military Health Care, retreating army, Maestrazgo Battle.

\section{INTRODUCCIÓN: ESTADO DE LA CUESTIÓN. CONTEXTO BÉLICO}

La historiografía sobre la Guerra Civil española ha abarcado numerosos campos de estudio (la retaguardia republicana, el anticlericalismo, la represión, el exilio, etc.). A pesar de todo, la guerra sigue planteando lagunas significativas, como la economía, la propaganda y el patrimonio bélico ${ }^{1}$. A esta lista de lagunas deseamos añadir la sanidad, especialmente en el ámbito local valenciano; efectivamente, Girona y Santacreu en su estado de la cuestión citan un total de 21 trabajos locales y comarcales realizados entre 1986 y $2006^{2}$; sin embargo, en esta lista tan solo se aprecia uno referido a la sanidad ${ }^{3}$, aunque hay que señalar que se obvia el trabajo de Cardona ${ }^{4}$. Con

${ }^{1}$ Doctor en Medicina y Cirugía. Universidad de Valencia. Valencia. España.

Dirección para correspondencia: xagarfe@alumni.uv.es

Recibido: 9 de febrero de 2012

Aceptado: 21 de junio de 2012 posterioridad han sido publicados otros trabajos centrados en el ámbito de la sanidad valenciana durante la Guerra Civil ${ }^{5-13}$.

La presente investigación realiza una aproximación a la Sanidad Militar del Ejército de Maniobra de la república en su retirada por la provincia de Castellón, y tiene sus antecedentes historiográficos inmediatos en esta revista ${ }^{14}$

Para entender el desarrollo de los acontecimientos se hace necesaria una breve contextualización histórica. A mediados del año 1937 el Estado Mayor republicano temía un ataque sobre Guadalajara, que acabaría por rodear Madrid por completo. Para evitarlo se planeó una maniobra de distracción, la conquista de Teruel. Así, el 15 de diciembre de 1937, bajo una fuerte nevada y con temperaturas de 16-18 grados bajo cero, 100.000 hombres se pusieron en marcha hacia Teruel. La ofensiva terminó el 7 de enero de 1938 con la ciudad tomada por las fuerzas republicanas y numerosas bajas en ambos bandos por la intensidad de los combates y por el frío. Sin embargo, diez días después se pusieron en marcha los Cuerpos de Ejército de los generales Aranda y Varela que, con una gran cobertura aérea y de artillería, lograron derrotar al Ejército Popular el día 
7 de febrero de 1938 en la batalla de Alfambra. El día 20 de febrero las fuerzas republicanas abandonaron definitivamente Teruel.

Después de la reconquista de Teruel, el Ejército de la república había quedado muy dañado. Aprovechando esta situación, el Estado Mayor franquista emprendió una ofensiva que tenía como objetivo llegar al mar Mediterráneo, partiendo así el territorio fiel a la República. La Batalla del Maestrazgo comenzó el 9 de marzo de 1938, y el día 15 de abril las tropas nacionales lograron su objetivo al llegar a Vinaròs (Castellón). El general Franco continuó esta potente ofensiva hacia el sur con el objetivo de ocupar Valencia, lo que supuso la retirada paulatina del Ejército de Maniobra en dirección sur por toda la provincia de Castellón.

La Batalla del Maestrazgo se caracterizó por el rápido y contundente avance de las tropas franquistas en un frente muy amplio. Finalmente el frente se estabilizó a lo largo de la línea comprendida entre las localidades castellonenses de Viver (interior) y Nules (costa), donde el Ejército de la república había articulado un sistema defensivo conocido como la línea XYZ. Además, en plena ofensiva, las tropas franquistas recibieron la orden de desplazar el máximo número de efectivos hacia el norte, para hacer frente a la ofensiva republicana del Ebro ${ }^{15}$.

\section{LA ACTUACIÓN DEL CAPITÁN MÉDICO DURANTE LA BATALLA DEL MAESTRAZGO}

El objetivo de este apartado es aproximarse a los servicios sanitarios del Ejército de Maniobra durante la Batalla del Maestrazgo (abril-julio de 1938); concretamente, analizaremos las características y la trayectoria de un hospital de primera línea a través del testimonio oral de su máximo responsable, un Capitán Médico republicano. Las entrevistas tuvieron lugar en diciembre de 2009, y el entrevistado mostró su deseo de mantenerse en el anonimato, por lo que nos referiremos a él a través de sus inciales.

Don P.I.M (Valencia, 1915-2011) estudiaba Medicina cuando la insurrección militar del 18 de julio de 1936 le sorprendió recién acabado el quinto curso. El otoño de aquel año se presentó voluntario al Ejército y su primer destino fue el cuartel de Benalúa (Alicante), donde estaba formándose la $44^{\mathrm{a}}$ Brigada Mixta. La Navidad de 1936, tras la instrucción, obtuvo el grado de capitán; asimismo,

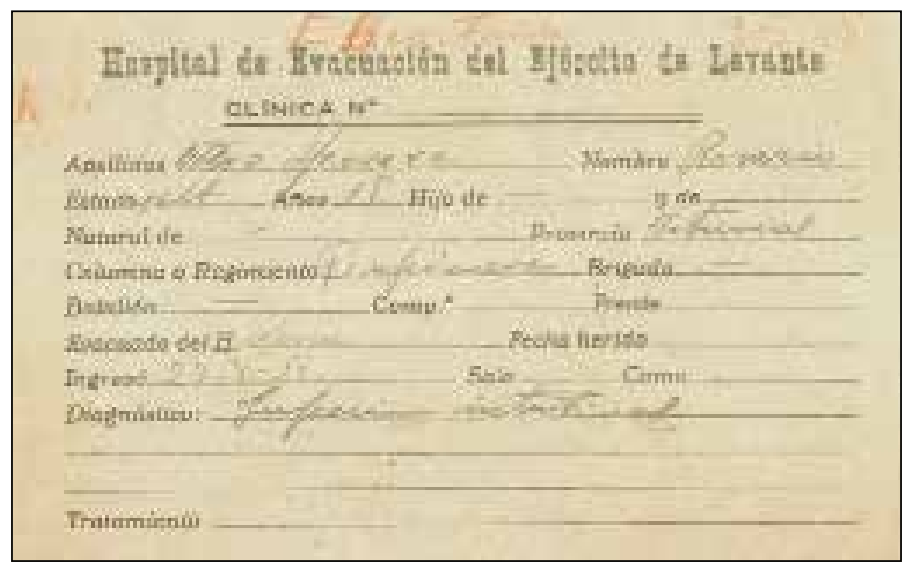

Figura 1. En el verano de 1938 el Hospital del Ejército de Levante contaba dos clínicas propias en Valencia. Fuente: A.D.P.V., I-2.4 c. 12, l. 46 . recibió la habilitación para ejercer la medicina y fue destinado, junto con otros médicos valencianos, a la defensa de Madrid. Al llegar a la capital, P.I.M se encontró en medio de una gran desorganización militar, especialmente de la Sanidad, por lo que se dirigió a la Jefatura de Sanidad del Ejército del Centro y solicitó hablar con José Estellés Salarich ${ }^{16}$. Finalmente, P.I.M. habló con Julio González Recatero, mano derecha de Estellés, a quien expuso la falta de organización y liderazgo sanitario de su brigada. Esta entrevista tuvo sus consecuencias, ya que unos días después los médicos valencianos recibieron la orden de incorporarse a los servicios médicos de la 44 a Brigada Mixta, que ya disponía de jefe de Sanidad. Se trataba de Carlos Díaz Fernández, médico que había sido responsable de la lucha antituberculosa en Madrid y que al estallar la guerra se había militarizado, obteniendo el grado de comandante. Díaz Fernández colocó a cada uno de los médicos valencianos al frente de un puesto sanitario de batallón excepto a P.I.M, a quien nombró su secretario particular. Juntos organizaron los servicios sanitarios de la $44^{a}$ Brigada Mixta.

La labor del comandante Díaz Fernández y del capitán P.I.M fue muy eficaz, de manera que el Estado Mayor republicano les encargó

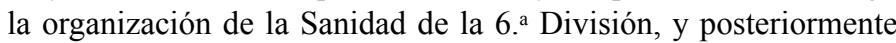
del 3. ${ }^{\text {er }}$ Cuerpo de Ejército republicano ${ }^{17}$. Este Cuerpo de Ejército cubría un frente de más de 20 kilómetros desde la Ciudad Universitaria a Las Rozas pasando por El Pardo; de hecho, el Estado Mayor republicano y la Jefatura de Sanidad del Ejército del Centro estaban instalados en el Palacio de El Pardo. Asimismo, en el Palacio de la Zarzuela, situado cerca de la primera línea de combate, había instalado un puesto sanitario. La misión del capitán P.I.M era recorrer todas las infraestructuras sanitarias de este amplio frente para coordinarlas, para comprobar que el funcionamiento era el correcto y para abastecerlas de material y personal.

Por tanto, podemos afirmar que fue en la defensa de Madrid donde P.I.M se forjó como experto en logística y gestión de infraestructuras sanitarias de guerra.

En agosto de 1937 P.I.M volvió a Valencia. Como hemos señalado, el Gobierno estaba reorganizando el Ejército de Maniobra para la ofensiva sobre Teruel; sin embargo, se dio cuenta de que faltaban médicos, por lo que el Ministerio de Defensa decidió abrir la Facultad de Medicina de Valencia para formar rápidamente médicos de guerra. P.I.M (recordemos que no había teminado la carrera) realizó este curso acelerado y antes de la Navidad de 1937 pasó a dirigir la Sección de Hospitales de la Jefatura de Sanidad del Ejército de Levante, que tenia su sede en el número 86 de la calle Colón de Valencia ${ }^{18}$. Allí esperó la orden de incorporación al Ejército de Maniobra. Conviene aclarar que durante la Batalla del Maestrazgo el Ejército de Maniobra absorbió al Ejército de Levante; sin embargo, cuando en julio de 1938 el frente se estabilizó prevaleció este último nombre.

Como se ha señalado con anterioridad, el Ejército de Maniobra había logrado tomar Teruel en enero de 1938, pero un mes después había sido derrotado y había comenzado a retirarse paulatinamente. En este contexto, a principios de abril de 1938, el capitán P.I.M. recibió la orden de incorporarse a la altura de Morella (Castellón); sin embargo, el dia 4 de abril de 1938 las tropas fraquistas entraron en la ciudad y solamente pudo llegar a Catí, un pueblo situado a unos $20 \mathrm{~km}$. al sur de Morella. Allí, en la iglesia de Catí, se puso nuevamente a las órdenes de Julio González Recatero, que en aquel momento era el jefe de Sanidad del Ejército de Maniobra. La iglesia 
del pueblo se había convertido en un improvisado hospital donde yacían centenares de heridos de los dos bandos procedentes de la batalla de Morella. En este escenario dantesco de sangre y gemidos, el capitán P.I.M. recibió la orden de González Recatero de formar un equipo médico para poner en funcionamiento uno de los hospitales de primera línea que iban a cubrir la asistencia sanitaria del Ejército de Maniobra. Siguiendo el único criterio de la intuición, P.I.M. eligió a los cirujanos Massons ${ }^{19}$ y Sánchez Martínez. Una vez formado el equipo médico, se les hizo entrega de toda la infraestructura que formaba un hospital de primera línea: un autochir (furgoneta-quirófano), un camión, un autobús, unas 20 camas y unas 30 personas entre personal sanitario, personal auxiliar (cocineros, electricistas, chóferes, encargados de montar y desmotar el hospital) y centinelas.

El primer lugar donde P.I.M. instaló el hospital que dirigía fue el convento de Sant Pau, en las afueras de Albocàsser (Castellón). El capitán eligió este lugar porque era un punto estratégico, ya que disponía de unas buenas instalaciones y estaba cerca de una vía de evacuación rápida hacia la retaguardia. El hospital contaba con dos equipos quirúrgicos, formado cada uno por un cirujano jefe (Massons y Sánchez Martínez respectivamente), un ayudante y un anestesista. Además, se disponía de un número indeterminado de practicantes y enfermeras.

José María Massons era un cirujano catalán que había introducido una técnica nueva de sutura de la cavidad abdominal. Esta técnica consistía en coser el abdomen en dos planos: el peritoneo se cosía con seda y las demás capas (músculo, aponeurosis y piel) eran cosidas de forma provisional con un alambre quirúrgico. Posteriormente, cuando la situación clínica del paciente había mejorado y se encontrada evacuado en un hospital de retaguardia, se cosían por planos las diferentes capas de la pared abdominal. Esta técnica se introdujo para ahorrar tiempo (unos veinte minutos aproximadamente), una variable fundamental en cirugía de guerra.

El frente estaba en Albocàsser, a tan sólo cuatro o cinco kilómetros, ya que estaba en disputa el control de un cerro, desde el cual se dominaba una amplia zona. Durante el día la aviación y la artillería franquista facilitaban la toma del pueblo, pero de noche era la infantería republicana la que, a golpe de bayoneta, reconquistaba las posiciones.

Esta inestabilidad del frente unido a la proximidad de los bombardeos hizo que, tras seis días aproximadamente, el capitán P.I.M tomara la decisión de trasladar el hospital hacia el sur. Así pues, el equipo de P.I.M. retrocedió hasta llegar a La Pelejana, una pequeña pedanía del municipio de la Vall d'Alba, situada a unos 20 kilómetros al sur de Albocàsser. El capitán eligió esta ubicación porque estaba lejos del frente y en un cruce de carreteras, circunstancias que permitían realizar evacuaciones frecuentes y seguras. Allí instalaron el hospital en la almazara del cacique del pueblo (figura 2). Como el autochir proporcionaba una cantidad de luz limitada, idearon un candil artesanal a partir de botes vacíos de leche condensada y gasas empapadas en aceite, que colocaron en el cabezal de cada cama. Durante el tiempo que permanecieron en La Pelejana, el personal del hospital llegó a confraternizar con la población de la comarca. Buena prueba de ello es que el hospital atendió a la población civil (por ejemplo, un parto y un aborto).

A los 20 días de estar allí, recibió la orden de evacuación porque el enemigo había avanzado y se encontraba muy cerca. Fue entonces cuando el capitán P.I.M. decidió instalar el hospital en una caseta de peones camineros situada en la carretera entre Vilafamés y Sant

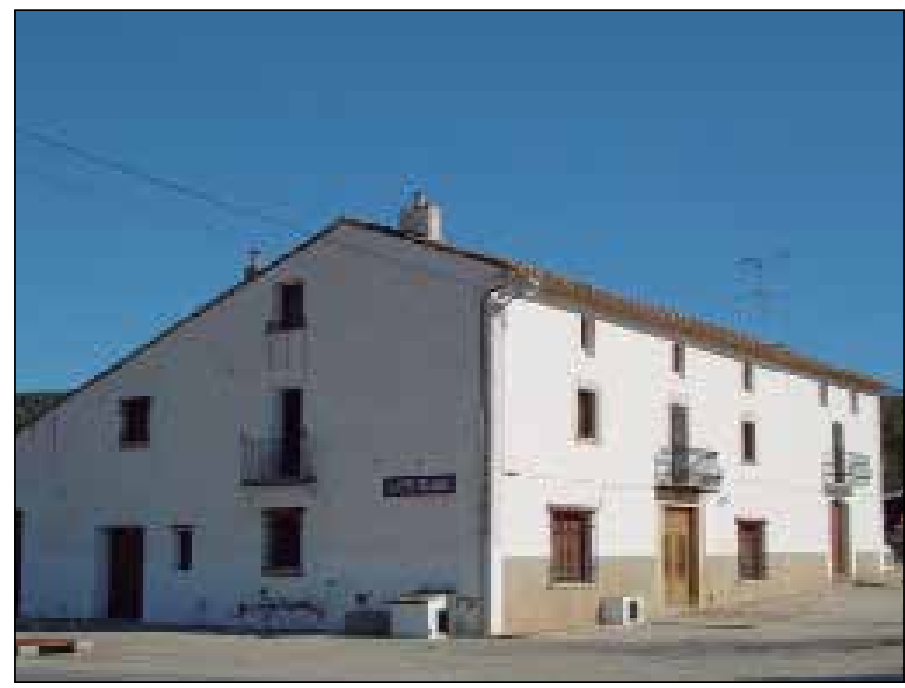

Figura 2. En la almazara de esta masía del Maestrazgo castellonense se instaló, durante la primavera de 1938, un hospital de primera línea. Fuente: el autor.

Joan de Moró, a unos 12 kilómetros al sur de La Pelejana. El lugar no era del todo del agrado de P.I.M. porque, aunque estaba en una carretera, ésta era transversal y no constituía una vía de evacuación rápida hacia Castellón; sin embargo, el capitán consideró que no había otro emplazamiento mejor y decidió desplegar el hospital de forma parcial (sólo se instalaron unas siete u ocho camas). Un día, durante la estancia en la caseta de peones camineros, P.I.M decidió buscar noticias del enemigo, ya que hacía tiempo que el Estado Mayor no le había informado sobre las posiciones de las tropas franquistas. Con este motivo, se subió a un cerro para ver si divisaba el enemigo. En ese momento comenzó un ataque de la aviación enemiga, que tenía como objetivo destruir la línea de fortificación que estaba construyendo el general Matallana. Este bombardeo obligó al capitán P.I.M. a permanecer tres horas agazapado en un boquete que había hecho uno de los proyectiles.

Al cabo de cuatro o cinco días, se recibió la orden de evacuar y retroceder de nuevo. Entonces P.I.M se vio envuelto en una encrucijada: en el hospital había un herido de cráneo muy grave que no podía ser trasladado. Tras haber esperado hasta el último momento, P.I.M. decidió abandonar el herido con una carta y un parte médico dirigidos a los servicios sanitarios del enemigo. Según nuestra fuente, esta práctica era habitual en la retirada de los ejércitos de ambos bandos.

En la figura 3 se puede observar una ficha médica utilizada durante la Batalla del Maestrazgo.

Después de hacer noche en Eslida (Castellón), el equipo finalmente llegó a Segorbe, que había sido hasta entonces una ciudad de retaguardia; sin embargo, el imparable avance de las tropas nacionales durante junio de 1938 había hecho que el frente se trasladase a las proximidades de Segorbe y que la ciudad fuera objeto de bombardeos. Lo primero que hizo P.I.M. al llegar al Hospital de Segorbe fue hacer entrega al Ejército del hospital de primera línea que había tenido a su mando. Posteriormente mantuvo una entrevista con el cirujano jefe del centro. Álvaro López era traumatólogo y antes de ser movilizado había sido director del Sanatorio Antituberculoso de la Malva-rosa (Valencia). Así pues, el joven capitán se vio en la tesitura de tener bajo sus órdenes a una eminencia 


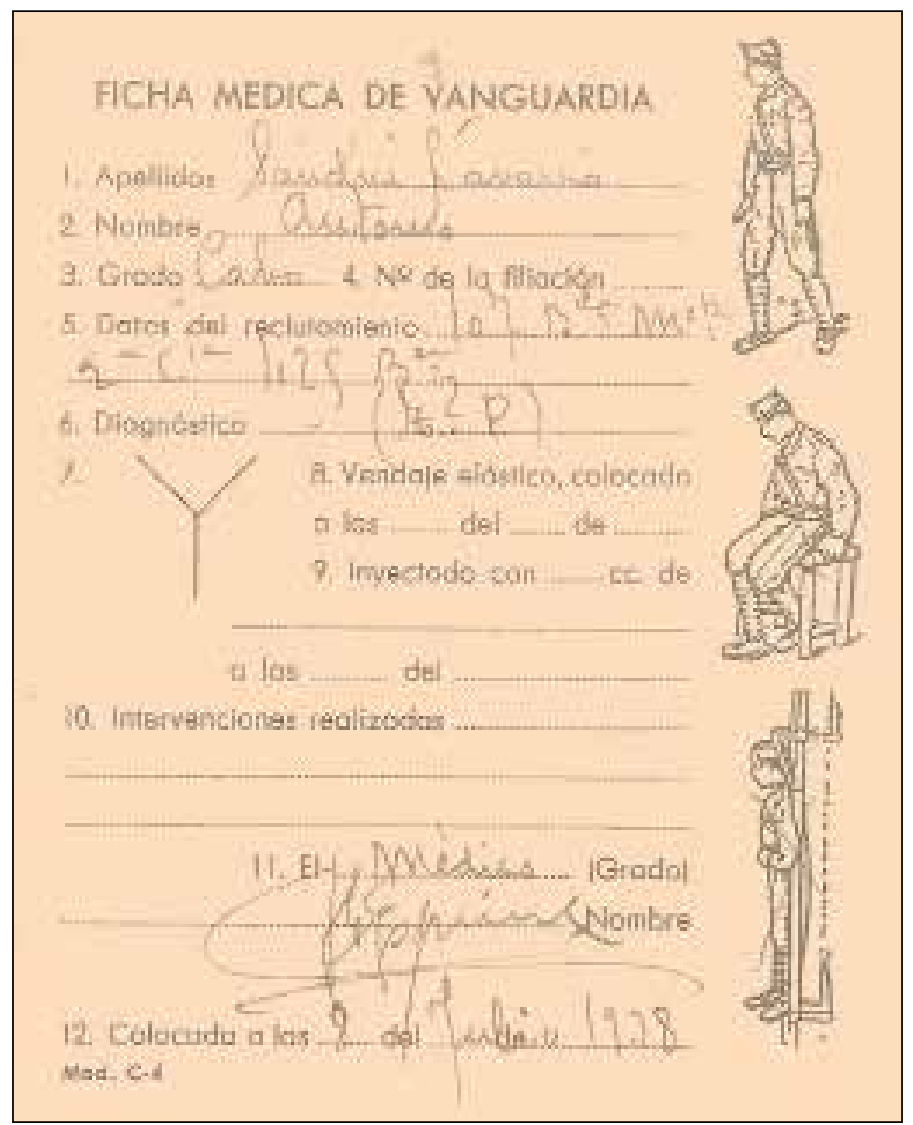

Figura 3. Ficha médica. Fuente: Archivo de la Diputación Provincial de Valencia, I-2.4 c. 12, legajo 45, "Ficha médica de vanguardia» con fecha 8 de julio de 1938.

reconocida de la cirugía valenciana. Sin embargo, el Hospital de Segorbe estaba militarizado, por lo que P.I.M. asumió la dirección del centro sanitario y tuvo bajo sus órdenes a los equipos de López y Bartrina. Bartrina había sustituido a Massons al ser éste destinado a otro hospital.

A los pocos días de la llegada a Segorbe P.I.M. fue testigo de un intenso bombardeo que provocó una masacre de civiles porque uno de los proyectiles cayó en la puerta de un refugio. Los continuos bombardeos y la proximidad del frente hizo que en el Hospital de Segorbe se llevara cabo una actividad quirúrgica frenética. En este sentido, los dos equipos de cirujanos operaban en dos quirófanos con cuatro mesas; así, cuando el cirujano jefe había acabado la parte más compleja de la operación, pasaba a otra mesa, donde le esperaba un segundo paciente anestesiado y preparado. Al mismo tiempo, los cirujanos ayudantes acababan de coser al primer herido.

Otra misión muy importante de P.I.M. en Segorbe fue organizar las evacuaciones de heridos a Valencia. Estos traslados se hacían en un tren sanitario, en concreto, en el tren sanitario número uno, ya que los servicios sanitarios del Ejército de la república disponían de una flota de seis trenes sanitarios ${ }^{19}$. El tren sanitario permanecía oculto en un túnel próximo a Segorbe y cuando se tenía que evacuar a un contingente de heridos, el capitán P.I.M. daba la orden de que el tren se situara en la estación; sin embargo, Segorbe es una ciudad construida en un cerro y la estación de ferrocarril está en la zona baja. Por tanto, en primer lugar había que trasladar a los heridos a la estación desde el hospital, que se hacía con ambulancias y con camillas. Durante una de estas evacuaciones se produjo un bombardeo sobre la ciudad que, aunque no estaba dirigido contra la caravana de evacuación, generó una situación de gran pánico y confusión entre la misma. El capitán P.I.M. -a quien el ataque sorprendió en el hospital- transmitió la orden de cuerpo a tierra sin abandonar a los heridos hasta que el bombardeo cesara.

Como hemos señalado con anterioridad, a finales de julio de 1938 el frente se estabilizó a lo largo de la linea comprendida entre las localidades de Nules y Viver, y P.I.M. fue trasladado a Valencia. Allí el jefe de Sanidad del Ejército de Levante -Julio González Recatero- dispuso que P.I.M. pasara de la Sección de Hospitales a la Sección de Personal. Su misión entonces fue recorrer el frente establecido en el sur de la provincia de Castellón para atender las necesidades sanitarias de todo el Ejército de Levante, visitando y coordinando los diferentes puestos sanitarios de batallón y hospitales de primera línea que había establecidos en este frente. Finalmente, en septiembre de 1938, P.I.M. pasó a las oficinas de la Jefatura de Sanidad, donde siguió en la sección de Personal. Su misión entonces consistió en distribuir la plantilla de médicos en las diferentes infraestructuras sanitarias del frente, relevando a los sanitarios del frente cuando consideraba oportuno. Asimismo, el capitán P.I.M. se encargaba de redactar los informes de los médicos movilizados para proponer un ascenso, una medalla o una sanción, según el comportamiento en el frente. En este puesto permaneció hasta el final de la guerra.

En el mapa (figura 4, elaboración propia a partir del testimonio oral) se puede apreciar la ruta que siguió el capitán P.I.M. a lo largo de la Batalla del Maestrazgo, entre los meses de abril y julio del año 1938. En esta ruta queda claramente reflejada la situación de constante retirada en que se encontraba el Ejército de Maniobra durante aquella campaña. Rodeados con círculos rojos aparecen los diferentes lugares donde P.I.M. decidió instalar el hospital que tenía a su cargo hasta su llegada a Segorbe. Asimismo, aparece señalada la ciudad de Valencia como último de destino del capitán durante la guerra.

\section{CONCLUSIONES}

El testimonio oral de un Capitán Médico republicano nos ha permitido realizar una aproximación a la organización de la Sanidad Militar del Ejército de Maniobra durante la Batalla del Maestrazgo (abril-julio de 1938). Así pues, podemos concluir que:

1. La Batalla del Maestrazgo se caracterizó por el rápido y contundente avance de las tropas nacionales en un frente muy amplio. Esta inestabilidad del frente obligó a la Sanidad del Ejército de Maniobra a replegarse continuamente, montando y desmontando rápidamente los puestos de clasificación y los hospitales de primera línea, que se caracterizaron, por tanto, por su extraordinaria movilidad, adaptabilidad y ubicuidad. Se establece, por tanto, una relación entre la inestabilidad del frente y la organización de la Sanidad Militar.

2. El criterio fundamental para desmontar un hospital de primera línea y retroceder era la inseguridad creada por el avance del frente de guerra, mientras que el criterio para volver a instalar un hospital de estas características era la presencia de una vía rápida de evacuación hacia Castellón o Segorbe.

3. Durante junio de 1938, el avance de las tropas franquistas fue de tal intensidad que hizo que el Hospital Militar de Segorbe dejara 
La cobertura de un ejército en retirada: actuación de un capitán médico durante la Batalla...

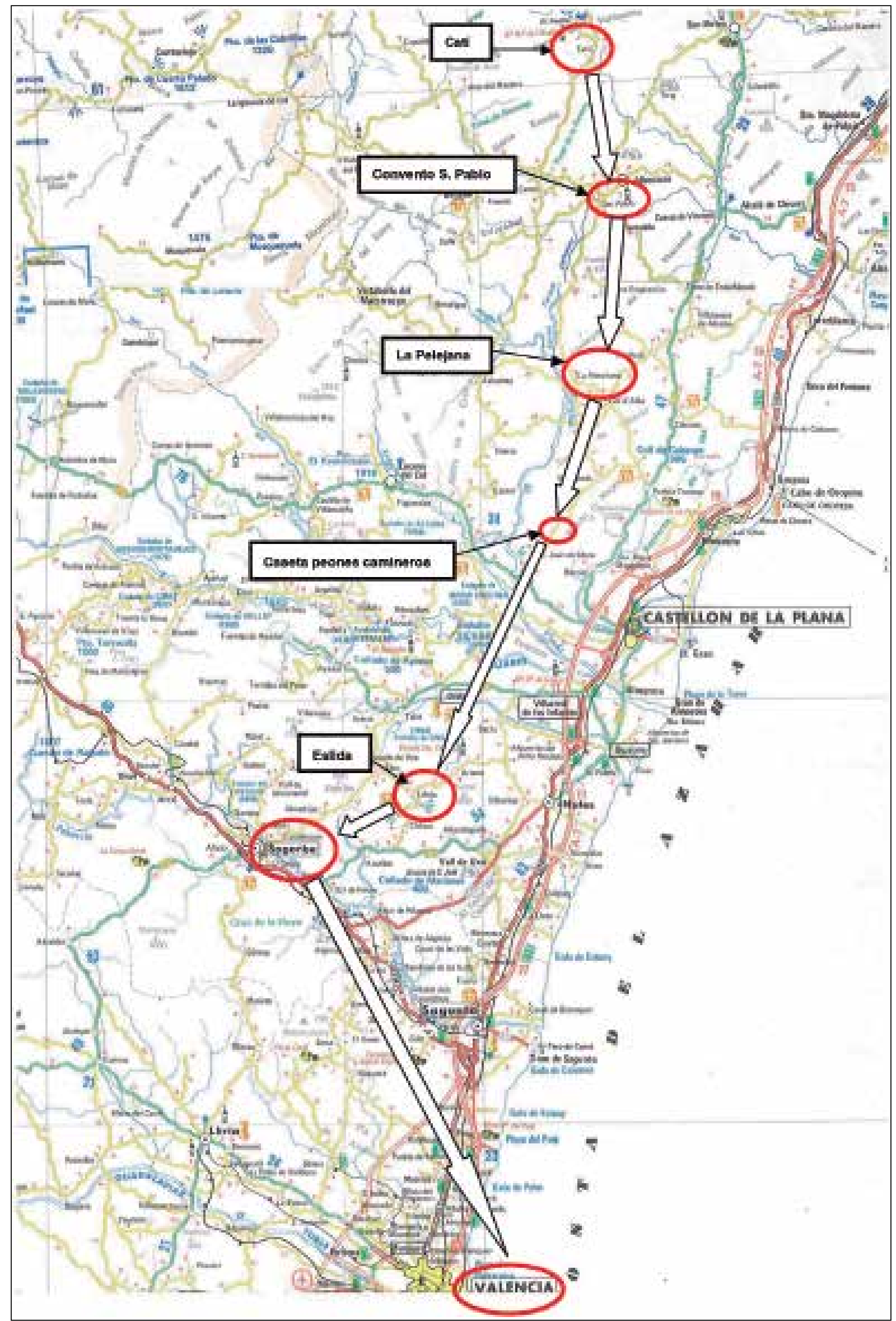

Figura 4. Ruta que siguió el capitán P.I.M. a lo largo de la Batalla del Maestrazgo. 
de actuar como hospital de retaguardia, es decir como centro receptor de heridos, para convertirse en un hospital de primera linea en toda regla que evacuaba heridos hacia los hospitales de retaguardia instalados en la ciudad de Valencia.

\section{AGRADECIMIENTOS}

\section{In memoriam}

A don P.I.M, Capitán Médico del Ejército de la república, por el coraje con que afrontó el momento histórico que le tocó vivir y por su impagable relato de aquel horror.

\section{BIBLIOGRAFÍA}

1. Calzado A, Girona A, Santacreu JM. La memoria de la Guerra Civil. En: Girona A, Santacreu JM (coords). La Guerra Civil en la Comunidad Valenciana. Vol. 18. Valencia: Editorial Prensa Valenciana, 2006: 21.

2. Ibid., 65 .

3. Beneito À. El hospital sueco-noruego de Alcoi durante la guerra civil española. Alcoi: Visual Producciones, 2004.

4. Cardona JJ. Hospitals militars a Benissa en la guerra de 1936. En: Bernabeu J, Esplugues JX, Robles E (eds). Higiene i Salubritat en els municipis valencians. Benissa: Institut de d'Estudis Comarcal de la Marina Alta, 1997: 223-225.

5. Barona JL. Salud, enfermedad y muerte. La sociedad valenciana entre 1833 y 1939.Valencia: Institució Alfons el Magnànim, 2002.

6. Casañ G. El hospital de Benicàssim en el contexto del servicio sanitario de las Brigadas Internacionales (Guerra Civil, 1936-1939). En: Requena M, Sepúlveda RM (coords) La sanidad en las Brigadas Internacionales. Cuenca: Ediciones de la Universidad de Castilla-La Mancha, 2006: 161-198.

7. Barona, JL. La sanitat en 1937. En: Aznar M (ed). València, capital cultural de la República. Vol. 2. Valencia: Consell Valencià de Cultura, 2007: 565-586.

8. Barona JL, Bernabeu J. Ciencia y sanidad en la Valencia capital de la República. Valencia: Publicacions de la Universitat de València, 2007.

9. Barona JL, Bernabeu J. La salud y el Estado. El movimiento sanitario internacional y la administración española (1851-1945). Valencia: Publicacions de la Universitat de Valencia, 2008.

10. García X. La reorganització de l'assistència medicoquirúrgica de la ciutat de València durant la Guerra Civil espanyola (1936-1939). Tesis doctoral inédita, Universitat de València, 2010.
11. García X. La asistencia sanitaria en la provincia de Valencia durante la Guerra Civil española (1936-1939). Llull 2011;34(73):13-38.

12. García X, García I. Itinerario didáctico por los hospitales de la Valencia en guerra. Didáctica de las Ciencias Experimentales y Sociales 2011; 25, 165-175.

13. García X. La asistencia psiquiátrica en la ciudad de Valencia durante la Guerra Civil española (1936-1939). Frenia. Revista de Historia de la Psiquiatría 2012;12 (en prensa).

14. García X, Munayco AJ. La asistencia sanitaria en el frente de Teruel durante la primera campaña republicana (agosto de 1936-febrero de 1937. Sanidad Militar 2010; 66 (4): 245-249; García X, Munayco AJ. La evolución de la Sanidad Militar en Valencia durante la Guera Civil Española (1936-1939). Sanidad Militar 2011; 67 (4): 385-391.

15. Massons JM. Historia de la Sanidad Militar Española Barcelona. Vol. 2. Ediciones Pomares-Corredor, 1994: 393-398.

16. Durante el primer bienio republicano (1931-1933), el médico valenciano José Estellés Salarich había ocupado la Secretaria General Técnica de la Dirección General de Sanidad bajo las órdenes del reformista Marcelino Pascua. Militarizado al estallar la guerra, llegó a dirigir la Sanidad del Ejército del Centro con el grado de comandante. Para más información sobre este médico consúltese: Bernabeu J. La salut pública que no va poder ser. José Estellés Salarich (1896-1990): una aportació valenciana a la sanitat espanyola contemporània. Valencia: Consell Valencià de Cultura, 2007.

17. La $44^{\mathrm{a}}$ Brigada Mixta pertenecía a la $6^{\mathrm{a}}$ División, que a su vez pertenecía al $3^{\text {er }}$ Cuerpo, circunstancia que permitió a P.I.M seguir en contacto con sus compañeros médicos valencianos.

18. Indicador de retaguardia activa: ministerios, centros oficiales, organizaciones políticas, económicas y sindicales, etc. etc. de Valencia. Valencia: Publicaciones Masenar, 1937: 34

19. Massons es el autor citado en la bibliografía de este trabajo. 